\title{
SYMMETRY-AXIS ELASTIC WAVES FOR TRANSVERSELY ISOTROPIC MEDIA*
}

\author{
$\mathrm{BY}$ \\ ROBERT G. PAYTON \\ Adelphi University
}

\begin{abstract}
An unbounded, linear transversely isotropic elastic solid is excited by a suddenly applied point body force of arbitrary orientation. Simple closed-form expressions are found for the various displacement components on the symmetry axis as a function of time and distance from the source. Applications are given for 13 hexagonal crystals.
\end{abstract}

Introduction. This paper treats the problem of the transient response of an unbounded, transversely isotropic, linear elastic solid, excited by a suddenly applied point body force. By restricting the analysis to the symmetry axis of the solid, it is possible to obtain explicit closed-form expressions for the various displacement compononts. These results constitute the first (three-dimensional, time-dependent) extension of Stokes' [1] celebrated solution for an isotropic solid to a (physically realizable) anisotropic solid.

Chee-Seng [2], who follows and extends the work of Lighthill [3], has analyzed the axial wave motion in transversely isotropic media with particular emphasis on a magnetohydrodynamic problem. The $w_{3}$ displacement component of the present paper conforms to the $P$ operator of Chee-Seng, but the $u_{1}$ component (due to the $\partial^{2} / \partial x^{2} M$ term) does not. Directly related to the present problem is the work of Cameron and Eason [4] who treated the displacement components in the transverse plane of the elastic solid, normal to the symmetry axis. Their results required numerical integration. The steady-state version of the above stated problem has been treated by Buchwald [5], who gave an asymptotic solution valid for the far field, based on the work of Lighthill [3].

The present paper is independent of the above-mentioned references. It follows and extends the two-dimensional wave propagation problem treated by the author [6]. The mathematical technique used for the multidimensional transform inversion in [6] is due to Burridge [7]. In the analysis contained below for radiation along the axis of material symmetry, the two-dimensional inversion technique of Burridge is extended to threedimensions. For a special combination of the elastic parameters, the $Q$ operator (defined in Sec. 2) factors into two wave operators with the result that the problem becomes much more tractable. This situation has been exploited by the author [8] and serves as a useful check on the present work.

In Sec. 2 the basic equations are stated and their solution is reduced to a residue calculation by means of integral transforms. The relevant pole locations are treated in Sec. 3 and then combined to give explicit expressions for the displacement components

\footnotetext{
* Received November 7, 1975.
} 
along the symmetry axis in Sec. 4 . These results are displayed graphically in Sec. 5 for thirteen representative hexagonal crystals.

2. Equations of motion and their solution. The equations of motion appropriate to a linear, transversely isotropic, elastic solid, acted upon by a time-dependent point body force, have been set down (in an uncoupled form) by the author in [9]. These equations are

$$
\begin{aligned}
L Q\left[u_{1}\right] & =-\left(Q+\frac{\partial^{2}}{\partial x^{2}} M\right)[\delta(\mathbf{x}) H(\tau)], \\
L Q\left[u_{2}\right] & =-\left(\frac{\partial^{2}}{\partial x \partial y} M\right)[\delta(\mathbf{x}) H(\tau)], \\
Q\left[u_{3}\right] & =\kappa\left(\frac{\partial^{2}}{\partial x \partial z}\right)[\delta(\mathbf{x}) H(\tau)], \\
Q\left[w_{3}\right] & =-(K)[\delta(\mathbf{x}) H(\tau)] .
\end{aligned}
$$

The various partial differential operators are defined by

$$
\begin{aligned}
& Q\left(\frac{\partial}{\partial x}, \frac{\partial}{\partial y}, \frac{\partial}{\partial z}, \frac{\partial}{\partial \tau}\right)=\alpha \frac{\partial^{4}}{\partial z^{4}}+\gamma \frac{\partial^{2}}{\partial z^{2}}\left(\frac{\partial^{2}}{\partial x^{2}}+\frac{\partial^{2}}{\partial y^{2}}\right)+\beta\left(\frac{\partial^{2}}{\partial x^{2}}+\frac{\partial^{2}}{\partial y^{2}}\right)^{2} \\
& -(\alpha+1) \frac{\partial^{4}}{\partial z^{2}} \frac{1 \tau^{2}}{2}-(\beta+1) \frac{\partial^{2}}{\partial \tau^{2}}\left(\frac{\partial^{2}}{\partial x^{2}}+\frac{\partial^{2}}{\partial y^{2}}\right)+\frac{\partial^{4}}{\partial \tau^{4}}, \\
& L\left(\frac{\partial}{\partial x}, \frac{\partial}{\partial y}, \frac{\partial}{\partial z}, \frac{\partial}{\partial \tau}\right)=\frac{\partial^{2}}{\partial z^{2}}+\delta\left(\frac{\partial^{2}}{\partial x^{2}}+\frac{\partial^{2}}{\partial y^{2}}\right)-\frac{\partial^{2}}{\partial \tau^{2}} \\
& M\left(\frac{\partial}{\partial x}, \frac{\partial}{\partial y}, \frac{\partial}{\partial z}, \frac{\partial}{\partial \tau}\right)=(1+\alpha \delta-\gamma) \frac{\partial^{2}}{\partial z^{2}}+(\delta-\beta)\left(\frac{\partial^{2}}{\partial x^{2}}+\frac{\partial^{2}}{\partial y^{2}}\right)-(\delta-\beta) \frac{\partial^{2}}{\partial \tau^{2}}, \\
& K\left(\frac{\partial}{\partial x}, \frac{\partial}{\partial y}, \frac{\partial}{\partial z}, \frac{\partial}{\partial \tau}\right)=\frac{\partial^{2}}{\partial z^{2}}+\beta\left(\frac{\partial^{2}}{\partial x^{2}}+\frac{\partial^{2}}{\partial y^{2}}\right)-\frac{\partial^{2}}{\partial \tau^{2}},
\end{aligned}
$$

where $x, y$ and $z$ are the Cartesian coordinates of a particle in the solid and the variable $\tau$ is related to the time coordinate $t$ by $\tau=\left(c_{44} / \rho\right)^{1 / 2} t, \rho$ being the mass density of the solid. As is evident from the structure of Eqs. (2.5)-(2.8), the $z$ axis is the symmetry axis of the solid. The five elasticity constants $c_{11}, c_{33}, c_{44}, c_{12}$ and $c_{13}$ have been combined into the dimensionless parameters

$$
\begin{aligned}
& \alpha=c_{33} / c_{44}, \quad \beta=c_{11} / c_{44}, \quad \gamma=1+\alpha \beta-\kappa^{2}, \\
& \delta=\frac{1}{2}\left(\beta-\left(c_{12} / c_{44}\right)\right), \quad \kappa=\left(\left(c_{13} / c_{44}\right)+1\right) .
\end{aligned}
$$

The point body force introduced into Eqs. (2.1)-(2.4) has the spatial dependence $\delta(\mathbf{x})=\delta(x) \delta(y) \delta(z)$, where $\delta(x)$ is the Dirac delta, and time dependence $H(\tau), H$ denoting the Heaviside unit step function. There are certain mathematical advantages gained by taking $H(\tau)$ rather than $\delta(\tau)$ for the time part of the source term, and in addition the static solution may be expected after a sufficient time interval has elapsed.

As for the displacement components, $u_{1}$ represents the $x$-component of displacement due to a body force acting in the $x$-direction, $u_{2}$ is the $x$-component of displacement due to a body force acting in the $y$-direction and $u_{3}$ is the $x$-component of displacement 
due to a body force acting in the $z$-direction. Similar statements hold for the $y$ and $z$-axis displacement components $v_{1}, v_{2}, v_{3}$ and $w_{1}, w_{2}, w_{3}$. Various symmetries allow the nine displacement components to be expressed in terms of the four quantities $u_{1}, u_{2}, u_{3}$ and $w_{3}$ since

$$
\begin{aligned}
v_{1}(x, y, z, \tau) & =u_{2}(x, y, z, \tau), \quad v_{2}(x, y, z, \tau)=u_{1}(y, x, z, \tau), \\
w_{1}(x, y, z, \tau) & =u_{3}(x, y, z, \tau), \quad v_{3}(x, y, z, \tau)=u_{3}(y, x, z, \tau), \\
w_{2}(x, y, z, \tau) & =v_{3}(x, y, z, \tau) .
\end{aligned}
$$

The central problem of this paper is the determination of the displacement components along the axis of rotational symmetry, as a function of the variables $z$ and $\tau$, for an unbounded elastic solid which is initially unstrained. The integration technique used in finding $w_{3}$ will now be briefly described.

By means of triple Fourier (in $x, y$ and $z$ ) and Laplace (in $\tau$ ) transforms, $w_{3}$ can be represented by the four-fold integral

$$
\begin{aligned}
w_{3}(x, y, z, \tau)=\frac{1}{2 \pi i} \int_{c} \exp (s \tau) d s \frac{1}{(2 \pi)^{3}} \int_{-\infty}^{+\infty} \int_{-\infty}^{+\infty} \int_{-\infty}^{+\infty} \frac{-K(i \xi, i \eta, i \zeta, s)}{s Q(i \xi, i \eta, i \zeta, s)} \\
\cdot \exp (i(\xi x+\eta y+\zeta z)) d \xi d \eta d \zeta .
\end{aligned}
$$

Introduce spherical coordinates $(R, \theta, \phi)$ by $\xi=R \sin \theta \cos \phi, \eta=R \sin \theta \sin \phi$ and $\zeta=R \cos \theta$; then

$$
\begin{aligned}
w_{3}(0,0, z, \tau) & =\operatorname{Re} \frac{1}{2 \pi i} \int_{c} \exp (s \tau) d s \frac{1}{(2 \pi)^{2}} \int_{0}^{\infty} R^{2} d R \\
\cdot & \int_{0}^{\pi}\left[\frac{-K(i R \sin \theta, 0, i R \cos \theta, s)}{s Q(i R \sin \theta, 0, i R \cos \theta, s)}\right] \times \exp (i R|z| \cos \theta) \sin \theta d \theta
\end{aligned}
$$

where special properties of the $K$ and $Q$ operators have been used (transverse isotropy), together with $x=y=0$, to perform the $\phi$ integration. Next set $s=\sigma R$ so that

$$
\begin{array}{r}
w_{3}=\operatorname{Re} \frac{1}{2 \pi i} \int_{c} \exp (\sigma R \tau) d \sigma \frac{1}{(2 \pi)^{2}} \int_{0}^{\infty} d R \int_{0}^{\pi} \sin \theta d \theta \frac{-K(i \sin \theta, 0, i \cos \theta, \sigma)}{\sigma Q(i \sin \theta, 0, i \cos \theta, \sigma)} \\
\cdot \exp (i R|z| \cos \theta) .
\end{array}
$$

At this stage the $\sigma$-integration can easily be performed followed by the $R$-integration (after suitably interpreting the $R$-divergent integral). Recombining the various terms in the integrand by partial fractions (in reverse) allows $w_{3}$ to be written as

$$
w_{3}=\operatorname{Re} \lim _{\epsilon \rightarrow 0+} \frac{i}{(2 \pi)^{2} \tau} \int_{0}^{\pi} \sin \theta d \theta \frac{K(\sin \theta, 0, \cos \theta, g)}{g Q(\sin \theta, 0, \cos \theta, g)},
$$

where $g(\theta)=|\bar{z}| \cos \theta+i \epsilon$ and $\bar{z}=z / \tau$. Following Burridge [7], the substitution $p=\cot \theta$ converts the integral (2.14) into

$$
w_{3}=\operatorname{Re} \lim _{\epsilon \rightarrow 0+} \frac{i}{(2 \pi)^{2}} \tau-\int_{-\infty}^{+\infty} \frac{K(1,0, p, h)}{h Q(1,0, p, h)} d p
$$

where $h=|\bar{z}| p+i \epsilon$. By closing the contour in the upper half $p$-plane, the integral (2.15) may be reduced to a residue calculation. The integrand of (2.15) contains two types of poles, those which remain in the upper half $p$-plane when $\epsilon=0$ and those which drift 
down onto the real axis as $\epsilon \rightarrow 0$. A method for locating the latter poles (i.e. real poles which are perturbed above the real axis when $\epsilon>0$ ) is given in Appendix $A$. The relevant real pole contributions to $w_{3}$ and $u_{1}$ are denoted by $w_{3}{ }^{*}$ and $u_{1}{ }^{*}$ in the formulas below.

$$
\begin{aligned}
w_{3}(0,0, z, \tau)=\frac{1}{2 \pi \tau} \operatorname{Re} \sum_{p}\left\{\text { residues of } \frac{-K(1,0, p,|\bar{z}| p)}{|\bar{z}| p Q(1,0, p,|\bar{z}| p)}\right. \\
\text { in the upper half } p \text {-plane }\}+w_{3}{ }^{*} .
\end{aligned}
$$

Using a similar argument, the other three displacement components defined by Eqs. (2.1)-(2.3) are given by

$$
\begin{gathered}
u_{1}(0,0, z, \tau)=\frac{1}{2 \pi \tau} \operatorname{Re} \sum_{p}\left\{\text { residues of }-\frac{Q(1,0, p,|\bar{z}| p)+M(1, p,|\bar{z}| p) / 2}{|\bar{z}| p L(1,0, p,|\bar{z}| p) Q(1,0, p,|\bar{z}| p)}\right. \\
\text { in the upper half } p \text {-plane }\}+u_{1}{ }^{*}, \\
\begin{array}{c}
\vdots \\
u_{2}(0,0, z, \tau) \equiv 0, \quad u_{3}(0,0, z, \tau) \equiv 0 .
\end{array}
\end{gathered}
$$

In view of Eqs. (2.18) and (2.10) it is seen that only the primary displacement components $u_{1}, v_{2}$ and $w_{3}$ differ from zero along the symmetry axis of a transversely isotropic solid subjected to an arbitrarily oriented point load on this axis.

The above details explaining the multiple transform inversion have necessarily been brief, but a fuller discussion (for an analogous two-dimensional problem) can be found in [6].

3. Location of the zeros of $f(p)$ in the upper half $p$-plane. From Eqs. (2.5)-(2.8) the $p$-dependence of the various terms in Eqs. (2.16) and (2.17) may be written

$$
\begin{aligned}
K(1,0, p,|\bar{z}| p) & =\left(1-\bar{z}^{2}\right) p^{2}+\beta \equiv g(p), \\
Q(1,0, p,|\bar{z}| p) & =\left(\alpha-\bar{z}^{2}\right)\left(1-\bar{z}^{2}\right) p^{4}+\left[\gamma-(\beta+1) \bar{z}^{2}\right] p^{2}+\beta \equiv f(p), \\
M(1,0, p,|\bar{z}| p) & =\left[(1+\alpha \delta-\gamma)-(\delta-\beta) \bar{z}^{2}\right] p^{2}+(\delta-\beta) \equiv m(p), \\
L(1,0, p,|\bar{z}| p) & =\left(1-\bar{z}^{2}\right) p^{2}+\delta \equiv l(p) .
\end{aligned}
$$

Of interest for the $w_{3}$ and $u_{1}$ residue calculations are the upper half $p$-plane zeros of $l(p)$ and $f(p)$. An examination of (3.4) shows that for $0 \leq|\bar{z}|<1$,

$$
l(p)=0 \quad \text { at } \quad p_{3}=i\left[\frac{\delta}{1-\bar{z}^{2}}\right]^{1 / 2},
$$

otherwise $\ell(\mathrm{p})$ has no upper half p-plane zeros.

Depending on the relative values of $\alpha, \beta, \gamma$ and $\bar{z}$, the biquadratic $f(p)=0$ may have 0,1 or 2 upper half $p$-plane roots. The real zeros of $f(p)$ can be found (and hence used to evaluate $w_{3}{ }^{*}$ and $u_{1}{ }^{*}$ ) from an analysis of the normal surface associated with the $Q$ operator. This is explained in Appendix $A$.

For all the hexagonal crystals listed in Sec. 5, the numerical values of the parameters $\alpha$ and $\beta$ satisfy

$$
\alpha>1, \quad \beta>1 .
$$

Assuming then that $\alpha$ and $\beta$ are both greater than one, the upper half $p$-plane zeros of $f(p)$ will now be catalogued as a function of $|\bar{z}|$ for various ranges of the parameter $\gamma$. 
$\frac{-2(\alpha \beta)^{1 / 2}<\gamma<(1+\alpha \beta)}{|\bar{z}| \geq \sqrt{ } \alpha}:$ no (relevant) real or complex roots,

$1<|\bar{z}|<\sqrt{ } \alpha$ :

$$
p=p_{1} \equiv i\left[\frac{-\left\{\gamma-(\beta+1) \bar{z}^{2}\right\}+D^{1 / 2}}{-2\left(\alpha-\bar{z}^{2}\right)\left(1-\bar{z}^{2}\right)}\right]^{1 / 2},
$$

$\frac{(\beta+1)<\gamma<(1+\alpha \beta)}{|\bar{z}|=1}:$

$$
p=p_{0} \equiv i\left[\frac{\beta}{\gamma-(\beta+1)}\right]^{1 / 2}
$$

$\frac{-2(\alpha \beta)^{1 / 2}<\gamma \leq(\beta+1)}{|\bar{z}|=1}:$ no (relevant) real or complex roots,

$\frac{(\alpha+\beta)<\gamma<(1+\alpha \beta)}{0 \leq|\bar{z}|<1}$ :

$$
\begin{aligned}
p & =p_{1} \quad \text { and } \quad p=p_{2} \\
& \equiv i\left[\frac{\left\{\gamma-(\beta+1) \bar{z}^{2}\right\}+D^{1 / 2}}{2\left(\alpha-\bar{z}^{2}\right)\left(1-\bar{z}^{2}\right)}\right]^{1 / 2},
\end{aligned}
$$

$\frac{(\beta+1)<\gamma<(\alpha+\beta)}{\bar{z}_{1}<|\bar{z}|<1}$

$$
\text { and }\left(\gamma^{2}-4 \alpha \beta\right)<0: \quad p=p_{1} \text { and } p=p_{2}
$$

$|\bar{z}|=\bar{z}_{1}:$

$p=p_{4} \equiv i\left[\frac{\beta}{\left(\alpha-z_{1}^{2}\right)\left(1-z_{1}^{2}\right)}\right]^{1 / 4}$

(double root),

$0 \leq|\bar{z}|<\bar{z}_{1}:$

$$
p=p_{+} \text {and } p=p_{-},
$$

where

$$
\begin{aligned}
& p_{ \pm} \equiv \pm\left[\frac{2\left\{\beta\left(\alpha-\bar{z}^{2}\right)\left(1-\bar{z}^{2}\right)\right\}^{1 / 2}-\left\{\gamma-(\beta+1) \bar{z}^{2}\right\}}{4\left(\alpha-\bar{z}^{2}\right)\left(1-z^{2}\right)}\right]^{1 / 2} \\
&+i\left[\frac{2\left\{\beta\left(\alpha-\bar{z}^{2}\right)\left(1-\bar{z}^{2}\right)\right\}^{1 / 2}+\left\{\gamma-(\beta+1) \bar{z}^{2}\right\}}{4\left(\alpha-\bar{z}^{2}\right)\left(1-\bar{z}^{2}\right)}\right]^{1 / 2}
\end{aligned}
$$

$\gamma<(\beta+1)$ and $\left(\gamma^{2}-4 \alpha \beta\right)<0$

$\bar{z}_{1}<|\bar{z}|<1 \quad p=p_{4}=\left[\frac{-\left\{\gamma-(\beta+1) \bar{z}^{2}\right\}+D^{1 / 2}}{2\left(\alpha-\bar{z}^{2}\right)\left(1-\bar{z}^{2}\right)}\right]^{1 / 2} \begin{aligned} & \text { and } p=-p_{4}, \text { no complex } \\ & \text { roots, }\end{aligned}$

$0 \leq|\bar{z}|<\bar{z}_{1}: \quad p=p_{+}$and $p=p_{-}$.

In the above expressions, $D$ is the discriminant of the biquadratic $f(p)$ and is given by

$$
\begin{aligned}
& D(\bar{z})=\left\{\gamma-(\beta+1) \bar{z}^{2}\right\}^{2}-4 \beta\left(\alpha-\bar{z}^{2}\right)\left(1-\bar{z}^{2}\right), \\
& D\left(\bar{z}_{1}\right)=0
\end{aligned}
$$

where

$$
\bar{z}_{1}=\left[\gamma(\beta+1)-2 \beta(\alpha+1)+2\{\beta(1+\alpha \beta-\gamma)(\alpha+\beta-\gamma)\}^{1 / 2}\right]^{1 / 2} /(\beta-1) .
$$

For the $\gamma$-range $\gamma<(\beta+1)$ and $\left(\gamma^{2}-4 \alpha \beta\right)<0, \bar{z}_{1}$ has an interesting physical interpretation. At the points $\bar{z}= \pm \bar{z}_{1} \tau$ on the $z$-zxis, the wave front surface associated with the $Q$ operator crosses itself and locally has the shape of a cone. Geometrically the normal 
curve $Q(s, 0, r,-1)=0$ (whose rotation about the symmetry axis generates the normal surface) has a real bitangent at $r^{-1}=\bar{z}_{1}$.

The above analysis of the complex roots of $f(p)=0$, while not exhaustive, covers all the materials listed in Sec. 5 and for this reason its further extention did not seem worthwhile. One notable omission is the case $\gamma=(\alpha+\beta)$, which has been treated in great detail [8]. This case includes the isotropic solid.

4. Residue calculation for $w_{3}$ and $u_{1}$. The residue calculations necessary to complete the formulas (2.16) and (2.17) for the axial displacement components $w_{3}$ and $u_{1}$ can now be easily performed, using the $p$-plane pole locations of the previous section. After some simplification, the final results are given by

$(\alpha+\beta)<\gamma<(1+\alpha \beta):$

$$
\begin{aligned}
& 0 \leq|\bar{z}| \leq 1, \quad 4 \pi|z| w_{3}(0,0, z, \tau)=1, \\
& 1 \leq|\bar{z}|<\sqrt{ } \alpha, \quad 4 \pi|z| w_{3}(0,0, z, \tau)=h(\bar{z}), \\
& \sqrt{ } \alpha \leq|\bar{z}|<\infty, \quad 4 \pi|z| w_{3}(0,0, z, \tau)=0,
\end{aligned}
$$

and

$$
\begin{array}{rlrl}
0 \leq|\bar{z}|<1, & 8 \pi|z| u_{1}(0,0, z, \tau) & =\frac{1}{\delta}+\frac{1}{\beta}, \\
1<|\bar{z}| \leq \sqrt{ } \alpha, & 8 \pi|z| u_{1}(0,0, z, \tau) & =k(\bar{z}), \\
\sqrt{ } \alpha & \leq|\bar{z}|<\infty, & 8 \pi|z| u_{1}(0,0, z, \tau) & =0 .
\end{array}
$$

$(\beta+1)<\gamma<(\alpha+\beta)$ and $\left(\gamma^{2}-4 \alpha \beta\right)<0$ :

Here the results are the same as those set down in Eqs. (4.1)-(4.6). $\gamma<(\beta+1)$ and $\left(\gamma^{2}-4 \alpha \beta\right)<0$ :

$$
\begin{array}{rlrl}
0 & \leq|\bar{z}|<\bar{z}_{1}, & & 4 \pi|z| w_{3}(0,0, z, \tau)=1, \\
\bar{z}_{1} & <|\bar{z}| \leq 1, & & \leq \pi|z| w_{3}(0,0, z, \tau)=2 h(\bar{z}), \\
1 & \leq|\bar{z}|<\sqrt{ } \alpha, & 4 \pi|z| w_{3}(0,0, z, \tau)=h(\bar{z}), \\
\sqrt{ } \alpha & \leq|\bar{z}|<\infty, & 4 \pi|z| w_{3}(0,0, z, \tau) & <0,
\end{array}
$$

and

$$
\begin{array}{rlrl}
0 \leq|\bar{z}|<\bar{z}_{1}, & 8 \pi|z| u_{1}(0,0, z, \tau) & =\frac{1}{\delta}+\frac{1}{\beta}, \\
\bar{z}_{1}<|\bar{z}|<1, & 8 \pi|z| u_{1}(0,0, z, \tau)=\frac{1}{\delta}+2 k(\bar{z}), \\
1<|\bar{z}| \leq \sqrt{ } \alpha, & 8 \pi|z| u_{1}(0,0, z, \tau)=k(\bar{z}), \\
\sqrt{ } \alpha \leq|\bar{z}|<\infty, & 8 \pi|z| u_{1}(0,0, z, \tau)=0 .
\end{array}
$$

The two new functions $h(\bar{z})$ and $k(\bar{z})$ introduced into the displacement expressions above are defined by

$$
h(\bar{z})=\frac{1}{2}-\frac{2\left(1-\bar{z}^{2}\right)-\left\{\gamma-(\beta+1) \bar{z}^{2}\right\}}{2 D^{1 / 2}},
$$




$$
k(\bar{z})=\frac{1}{2 \beta}+\frac{(\beta-1) \bar{z}^{2}+(\gamma-2 \alpha \beta)}{2 \beta D^{1 / 2}} .
$$

Both the functions $h$ and $k$ are defined on the interval $1<|\bar{z}|<\sqrt{ } \alpha$ and have the limit values

$$
\begin{gathered}
\lim _{|\bar{z}| \rightarrow 1+0} h(\bar{z})=1 \text { for } \gamma>(\beta+1) \\
=0 \text { for } \gamma<(\beta+1), \\
\lim _{|\bar{z}| \rightarrow \mid+0} k(\bar{z})=-\frac{1+\alpha \beta-\gamma}{\beta(\gamma-\beta-1)} \text { for } \gamma>(\beta+1) \\
=-\frac{\alpha-1}{\beta+1-\gamma} \text { for } \gamma<(\beta+1), \\
\lim _{|\bar{z}| \rightarrow \sqrt{\alpha-0}} h(\bar{z})=\frac{\alpha-1}{(\beta+1) \alpha-\gamma}, \\
\lim _{|\bar{z}| \rightarrow \vee_{\alpha-0}} k(\bar{z})=0 .
\end{gathered}
$$

The $w_{3}$ displacement component experiences jump discontiniuties at $|\bar{z}|=\sqrt{ } \alpha$ and at $|\bar{z}|=\bar{z}_{1}$, while $u_{1}$ has jump discontinuities at $|\bar{z}|=1$ and at $|\bar{z}|=\bar{z}_{1}$. Both displacement components are singular at $|\bar{z}|=\bar{z}_{1}$ when $\gamma<(\beta+1)$ and $\left(\gamma^{2}-4 \alpha \beta\right)<0$. This reciprocal square root singularity (due to the vanishing of $D\left(\bar{z}_{1}\right)$ ) is in agreement with Buchwald [5]. After passage of the trailing wave front, $|z|=\tau$ or $|z|=\bar{z}_{1} \tau$ as the case may be, the displacement components immediately assume their static (time-inde-

\begin{tabular}{|c|c|c|c|c|c|c|c|}
\hline Material & $\alpha$ & $\beta$ & $\gamma$ & $\delta$ & Case & $W_{+}$class & Remark \\
\hline Apatite & 2.11 & 2.52 & 2.34 & 1.16 & 4.9 & IV & $(3)$ \\
\hline Beryllium & 2.07 & 1.80 & 3.56 & 0.82 & 4.5 .1 & I & (2) \\
\hline Beryl & 3.62 & 4.11 & 11.81 & 1.33 & 4.13 .3 & $\mathrm{~V}$ & (1) \\
\hline Cadmium & 2.62 & 5.95 & 6.80 & 1.89 & 4.11 & II & (3) \\
\hline Cobalt & 4.74 & 4.07 & 14.69 & 0.94 & 4.5 .3 & $\mathrm{~V}$ & (1) \\
\hline Ice & 4.57 & 4.26 & 13.51 & 1.10 & 4.5 .3 & V & (1) \\
\hline Hafnium & 3.54 & 3.25 & 7.72 & 0.94 & 4.5 .1 & I & (1) \\
\hline Magnesium & 3.74 & 3.61 & 9.20 & 1.02 & 4.5 .1 & I & (1) \\
\hline Rhenium & 4.22 & 3.78 & 11.77 & 1.06 & 4.5 .3 & $\mathrm{~V}$ & (1) \\
\hline Titanium & 3.88 & 3.47 & 8.31 & 0.75 & 4.5 .1 & I & (1) \\
\hline Thallium & 7.27 & 5.62 & 16.93 & 0.37 & 4.5 .1 & I & (1) \\
\hline Yttrium & 3.16 & 3.20 & 7.81 & 1.00 & 4.13 .1 & I & (1) \\
\hline \multirow[t]{4}{*}{ Zinc } & 1.57 & 4.17 & 2.40 & 1.69 & 4.9 & IV & (3) \\
\hline & $(1$ & \multirow{3}{*}{\multicolumn{6}{|c|}{$\begin{array}{l}(\alpha+\beta)<\gamma<(1+\alpha \beta) \\
(\beta+1)<\gamma<(\alpha+\beta) \text { and }\left(\gamma^{2}-4 \alpha \beta\right)<0 \\
\gamma<(\beta+1) \text { and }\left(\gamma^{2}-4 \alpha \beta\right)<0 . \text { Also } \beta>\alpha\end{array}$}} \\
\hline & (2 & & & & & & \\
\hline & (3 & & & & & & \\
\hline
\end{tabular}
pendent) values. This deformation, without restriction to the symmetry axis, can of course be verified independently of the above analysis.

5. Application to some hexagonal crystals. In Table 1 the data of Hearmon [10] have been used to find the parameter values $\alpha, \beta, \gamma$ and $\delta$ for 13 hexagonal crystals. The "case" column refers to the previous classification of $[11,12]$, while the " $W_{+}$class"

TABLE 1. Elasticity constants for some hexagonal materials. 
classifies the inner sheet of the wave front associated with the $Q$ operator according to its shape.

Figs. 1-4 show the variation of the normalized displacement components $4 \pi|z| w_{3}(0,0, z, \tau)(\equiv w)$ and $8 \pi|z| u_{1}(0,0, z, \tau)(\equiv u)$ as a function of the single variable $\bar{z}$, for the crystals listed in Table 1 . The main features of these graphs have been anticipated in Sec. 4. In particular, apatite, cadmimu and zinc have singularities at the conical point on the wave front. The respective values of $\bar{z}_{1}$ for these materials are $0.9633,0.9997$ and 0.8871 . Cadmium is an interesting special case. Since $\gamma$ and $(\beta+1)$ are very close in numerical value, two inflection points on the wave front normal curve almost coalesce on the symmetry axis. This results in a large displacement as $\bar{z} \rightarrow 1+0$ which is extremely close to the singularity at $\bar{z}=\bar{z}_{1}$.

It is instructive to search for possible maximum and minimum values of the functions $h(\bar{z})$ and $k(\bar{z})$ defined by Eqs. (4.15) and (4.16). Now

$$
d h / d \bar{z}=-2(\alpha \beta+1-\gamma) \frac{\bar{z}\left[(\gamma-2 \beta)+(\beta-1) \bar{z}^{2}\right]}{D^{3 / 2}} .
$$

Under suitable conditions this slope will change sign in the interval $1<\bar{z}<\sqrt{ } \alpha$ with the result that $h$ takes on a maximum value

$$
h_{\max }=\frac{1}{2}\left[1-\left\{\frac{(\beta+1-\gamma)(\beta-1)}{(\beta+\alpha-\gamma) \beta}\right\}^{1 / 2}\right] \text { at }|\bar{z}|=\left[\frac{2 \beta-\gamma}{\beta-1}\right]^{1 / 2} .
$$

This is the situation for apatite and cadmium; for the other eleven crystals $h(\bar{z})$ is monotonic.

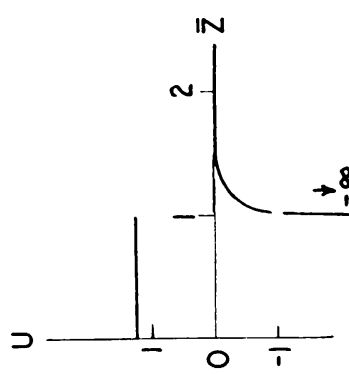

区

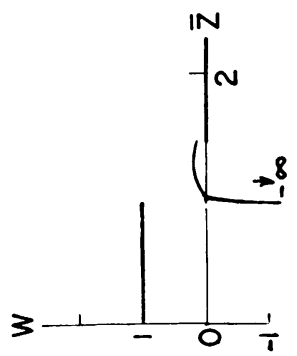

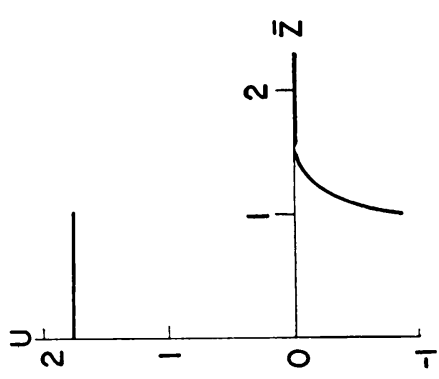

$\underline{\underline{0}}$

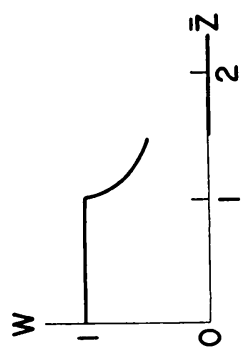

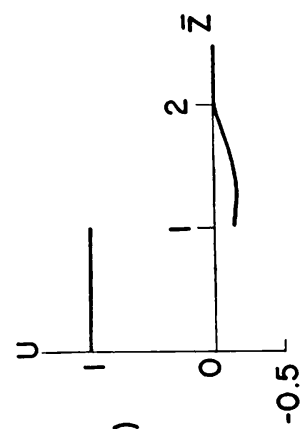

ভ̄

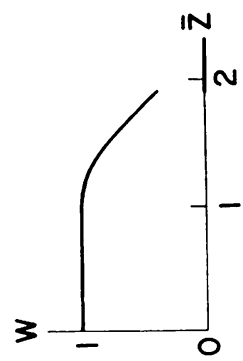

Fig. 1. Normalized displacement components $w$ and $u$ versus $\bar{z}$ for (A) apatite, (B) beryllium and (C) beryl. 

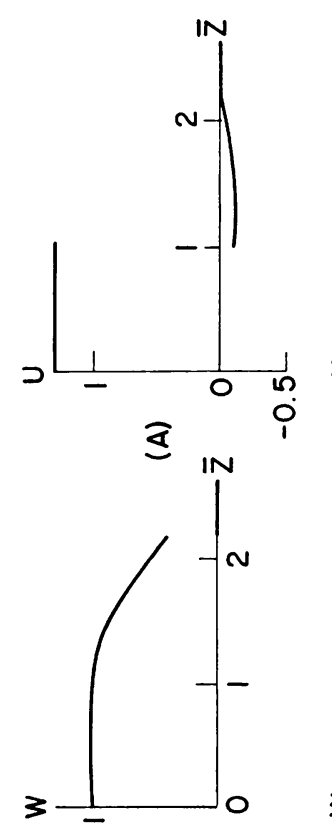

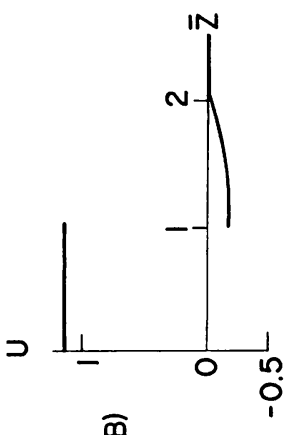

ติ

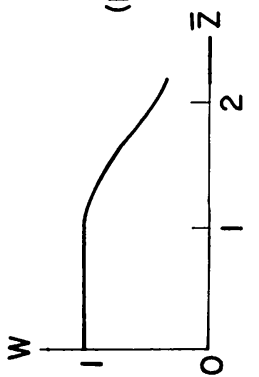

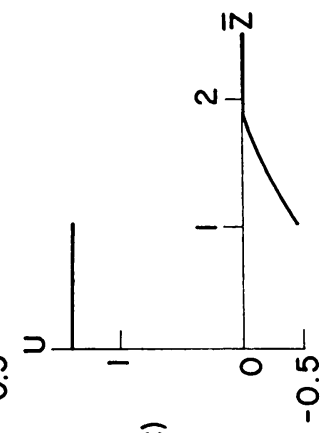

$\widehat{U}$

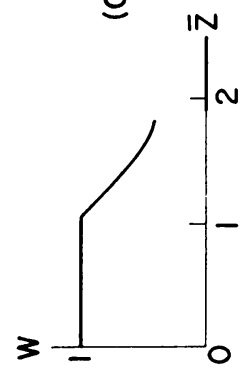

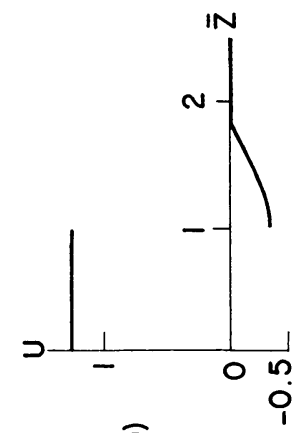

อิ

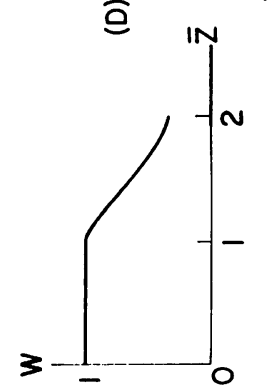

FIG. 2. Normalized displacement components $w$ and $u$ versus $\bar{z}$ for (A) cobalt, (B) ice, (C) hafnium and (D) magnesium.
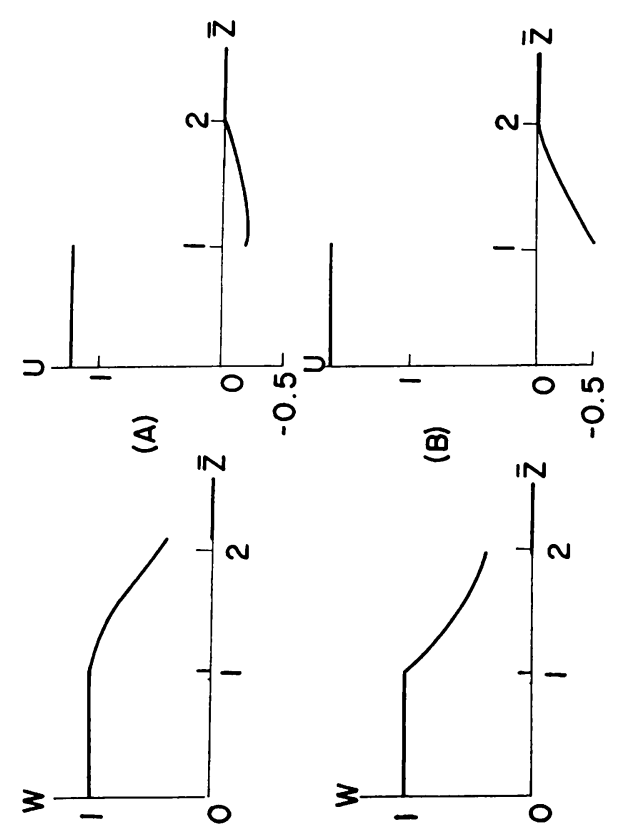

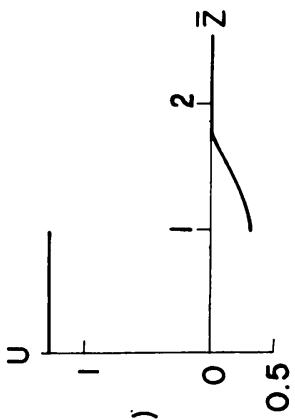

తิ

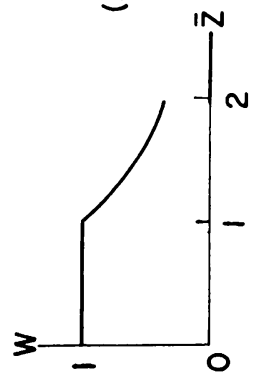

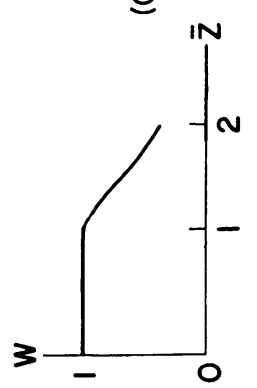

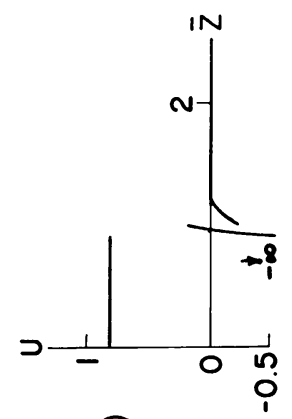

อ

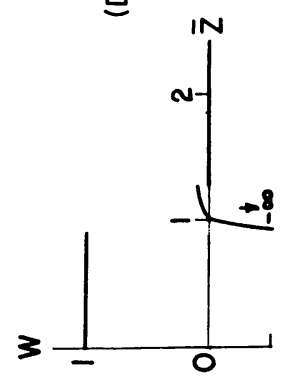

FIG. 3. Normalized displacement components $w$ and $u$ versus $\bar{z}$ for (A) rhenium, (B) titanium, (C) yttrium and (D) zinc. 


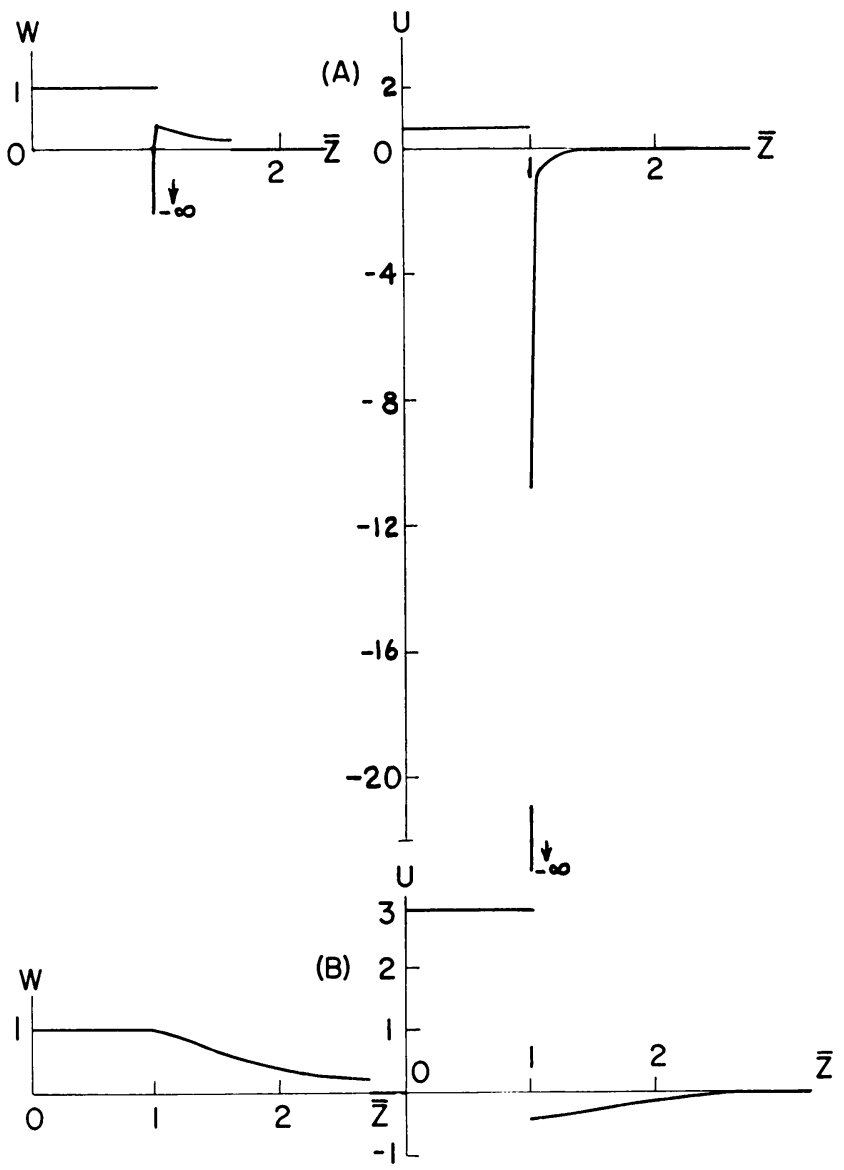

Fig. 4. Normalized displacement components $w$ and $u$ versus $\bar{z}$ for (A) cadmium and (B) thallium.

Similarly

$$
d k / d \bar{z}=-2(\alpha \beta+1-\gamma) \frac{\bar{z}\left[(\gamma-2 \alpha)-(\beta-1) \bar{z}^{2}\right]}{D^{3 / 2}},
$$

which under suitable conditions will give rise to a minimum value

$$
k_{\text {min }}=\frac{1}{2 \beta}\left[1-\left\{\frac{(\alpha \beta+\alpha-\gamma)(\beta-1)}{\gamma-\alpha-\beta}\right\}^{1 / 2}\right] \text { at }|\bar{z}|=\left[\frac{\gamma-2 \alpha}{\beta-1}\right]^{1 / 2} \text {. }
$$

This is the situation for beryl, cobalt, ice and rhenium; for the other nine crystals $k(\bar{z})$ is monotonic.

Acknowledgements. In an earlier version of this paper, a referee noted a mistake in that the displacements were nonsingular at $\bar{z}=\bar{z}_{1}$. The resolution of this difficulty, by including the appropriate real poles in the residue expressions, was pointed out to the author by Professor R. Burridge (private communication). 


\section{REFERENCES}

[1] G. G. Stokes, Trans. Camb. Philos. Soc. 9 (1851)

[2] L. Chee-Seng, Proc. Camb. Philos. Soc. 74369 (1973)

[3] M. J. Lighthill, Phil. Trans. Roy. Soc. London A 252397 (1960)

[4] N. Cameron and G. Eason, Quart. J. Mech. Appl. Math. 20, 23 (1967)

[5] V. T. Buchwald, Proc. Roy. Soc. London A 253, 563 (1959)

[6] R. G. Payton, Proc. Camb. Philos. Soc. 70, 191 (1971)

[7] R. Burridge, Proc. Camb. Philos. Soc. 63, 819 (1967)

[8] R. G. Payton, Quart. J. Mech. Appl. Math. 28, 473 (1975)

[9] R. G. Payton, Instituto Lombardo (Rend. Sc.) A 108, 684 (1974)

[10] Landolt-Börstein, Numerical data and functional relationships in science and technology. New Series, Group 3, vol. I, article by R. F. S. Hearmon. Berlin-Heidelberg-New York, Springer, 1969

[11] R. G. Payton, Arch. Rat. Mech. Anal. 32, 311 (1969)

[12] R. G. Payton, Arch. Rat. Mech. Anal. 35, 402 (1969)

Appendix A. Location of real poles which contribute to $w_{3}{ }^{*}$ and $u_{1}{ }^{*}$. Suppose that $p_{0}$ is a real root of $Q(1,0, p,|\bar{z}| p)=0$. Then the corresponding root of $Q(1,0, p,|\bar{z}| p+$ $i \epsilon)=0$ will be $p=p_{0}+i \epsilon p_{1}+\cdots$. The main question is the sign of $p_{1}$. By expanding $Q$ in a Taylor series in $\epsilon$, the value of $p_{1}$ is found to be $p_{1}=-\left[Q_{u} / Q_{v}+|\bar{z}|\right]^{-1}$ where $Q_{u}=(\partial / \partial u) Q(1,0, u, v), Q_{v}=(\partial / \partial v) Q(1,0, u, v)$ and $u$ and $v$ are to be replaced by $p_{0}$ and $|\bar{z}| p_{0}$ respectively after differentiation.

The curve $M: Q(1,0, u, v)=0$ in the $u, v$ plane has the slope $d v / d u=-Q_{u} / Q_{v}$. Geometrically the sign of $p_{1}$ is determined by (slope of line $v=|\bar{z}| u$ ) - (slope of curve $M$ ). If this quantity is negative, then the corresponding $p$-pole (for $\epsilon>0$ ) lies in the upper half $p$-plane and hence contributes to the residue calculation. Also, since $Q_{u} / Q_{v}$ is even in $p_{0}$, if the real pole $p_{0}$ contributes to the residue then so does the pole- $p_{0}$. For this reason only the first quadrant of the $u, v$ plane need be considered.

From the shape of the $M$-curve it is clear that the key question is the existence of a point on the curve where the (curve slope) $=$ (slope of the line $v=|\bar{z}| u)$. Such a point corresponds to a conical point on the symmetry axis of the wave front and corresponds to a bitangent on the normal (slowness) curve. If there is no such point (for the present problem) there will be no residue contribution from the real poles. For the crystals considered herein, only apatite, cadmium and zinc have real poles (for $\epsilon=0$ ) which are perturked to the upper half $p$-plane for $\epsilon>0$. The relevant $p$-poles are $p=p_{4}$ and $p=-p_{\mathbf{4}}$, as given in Eq. (3.16). 